\title{
EL MITO DEL ENFERMO MENTAL A TRAVÉS DEL ANÁLISIS DE TEXTOS
}

\author{
Rafael Pascual Martínez*; \\ Inmaculada Ortiz Viudes*
}

*D.E.Asistencial. Especialista en Enfermería en Salud Mental y Psiquiatría.

Correspondencia: Rafael Pascual Martínez.C/ Primavera n ${ }^{\circ} 18,2^{\circ}$ D. 03012 Alicante.

" la gente lo ignora todo sobre los indios, excepto lo poco que han visto en las películas"

KEN KESEY, "Alguien voló sobre el nido del cuco"

\section{RESUMEN}

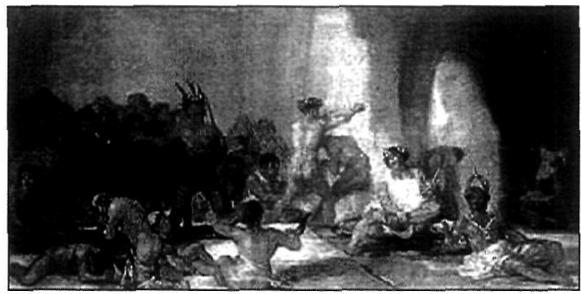

Se plantea la hipótesis de que la imagen negativa que la sociedad tiene del enfermo mental está reforzada por mensajes negativos y/o erróneos. MATERIAL Y MÉTODOS: Se procede al análisis de los mensajes contenidos en cuatro novelas con unas características comunes y definidas. DISCUSIÓN Y CONCLUSIONES: Destaca la visión negativa que del enfermo mental y de su entorno muestra cada uno de los libros, demostrándose la hipótesis inicial.

\section{THE MYTH OF THE MENTALLY IMPAIRED PATIENT THROUGH TEXT}

\author{
SUMMARY
}

\section{Introduction:}

An hypothesis of the negative image which society has of the mentally-impaired patient emphasised or reinforced by negative and false messages, is here presented. The contents of four novels with common and well-defined topics are discussed. The negative view each novel gives of $<$ mentally $>$-impaired patients is also regarded. Some conclussions are given. 


\section{INTRODUCCIÓN}

No hace ahora mucho tiempo, se emitió por televisión una película en la que un psiquiatra equipado de una jeringa con una aguja intravenosa del tamaño le un bolígrafo se disponía a realizar una exploración a un niño amarrado con correas a una camilla. Con in primer plano de la descomunal aguja, la voz del galeno tranquiliza al muchacho informándole de que an sólo es una inyección de "glucosa radioactiva". Afortunadamente consigue escapar, dejando atrás las mágenes de lo que se le avecinaba: un paciente atado a una silla con un sombrero eléctrico en su cabeza, una señora de la limpieza apartando del pasillo a los pacientes que se retorcían en el suelo... Para quien no le resulte ajeno el ambiente hospitalario en Psiquiatría estas imágenes podrían resultar bastante graciosas aunque, una vez analizado, el efecto que pretendían conseguir aquellas escenas era bien distinto. Lamentablemente, la emisión de contenidos utilizando la imagen del enfermo mental es más fresuente de lo que debiera, ya que lo que se consigue ss hacer un flaco favor a este colectivo de pacientes a través de la generalización y mitificación de determinados estereotipos.

En este sentido se expresa Mauleón García (1993) al afirmar que la población adopta unas actitudes muy ricas en creencias y estereotipos que no están suficientemente fundamentadas por la experiencia, predisposiciones que adolecen de falta de objetividad y apoyadas en una generalización excesiva. La actitud supone una predisposición a reaccionar frente a un problema y a una toma de posición que viene marcada emocionalmente por un componente afectivo resultado de una experiencia adquirida en el orden cultural (Marty y Murcia, 1988: 128)

En el terreno que nos ocupa, son diversos los autores que manifiestan la existencia de actitudes poco favorables hacia el enfermo mental y su entorno tanto en la población en general (Mauleón, 1993; Martí y Murcia, 1988: 128-133; Rubio, 1994; Cabrera, 1995; Molina et al. 1994; Ortiz et al.: 1995) como en profesionales de Enfermería (Mauleón, 1993). Estas actitudes negativas toman su importancia cuando se convierten en un obstáculo para la reinserción o la satisfacción de las necesidades socio- sanitarias de la persona enferma. Rubio (1994) afirma que las actitudes sociales condicionan la forma de dar atención, al igual que Cabrera (1995), para quien el halo de misterio e ignorancia que rodea la esquizofrenia produce que las estructuras sanitarias hagan oídos sordos en muchas ocasiones a las demandas de este colectivo.

Una muestra de la influencia que poseen las actitudes de la población sobre la posibilidad de integración de un determinado colectivo lo podemos encontrar en el caso del SIDA: de una etapa de marginación (incluso se boicoteaba la entrada de niños afectados a las escuelas) se ha pasado, gracias a las campañas de información y concienciación, a una nueva fase en la que la solidaridad y la integración están en auge. Una explicación de este cambio en el modo de pensar y actuar está en el hecho de que el hombre aprende e incorpora conocimientos, habilidades y experiencias sobre la salud, la enfermedad y la forma de autocuidarse a través de mensajes emitidos por diferentes fuentes (Isla et al., 1995: 59).

Para concluir con el estado de la cuestión, mencionar que no se ha hallado ningún trabajo que trate el tema siguiendo la metodología empleada (el análisis de textos), aunque autores como Hereile et al. (1992) y Siles et al. (1993) lo utilizaron con resultados satisfactorios. Esta primera destaca el impacto global de inseguridad y temor que ejerce la prensa sobre el lector al tratar un tema relacionado con la salud, llegando incluso a publicar noticias erróneas.

Una vez delimitado el problema que fundamenta la realización de este trabajo, se plantea como hipótesis que la imagen negativa que la sociedad tiene del enfermo mental está reforzada por mensajes negativos y/ o erróneos. El objetivo será la realización de un análisis de los mensajes que sobre el enfermo mental y su entorno se emite desde un determinado medio de comunicación, para determinar su posible vinculación con la generación de actitudes desfavorables. Como fuente de información para el análisis e interpretación de datos se utilizarán diversas novelas y textos narrativos que tengan como protagonistas a pacientes psiquiátricos. El segundo objetivo lo constituirá la evaluación del uso de textos narrativos como método alternativo de investigación

\section{El enfermo mental como figura literaria}

Pons Bertrán (1973) (Martí y Murcia, 1988: 129) considera fuentes de información sobre la imagen del enfermo mental elementos tan diversos como los medios de comunicación cuando tratan temas relacionados con la Psiquiatría; chascarrillos, refranes y 
chistes; la Antropología y la Sociología; y el conocimiento por parte de la población del lenguaje científico y el uso que hace de él. Otro de los medios de transmisión de información a los que hace referencia es el que sirve de objeto en el presente trabajo: la literatura de cada pueblo, que refleja la idea que éste tiene sobre los trastornos psíquicos.

Esto último es fácilmente comprobable si se hace una pequeña revisión de esta literatura a lo largo de la historia, y observamos como ésta ha venido reflejando no sólo la imagen del enfermo, sino también la terapéutica empleada. Así, ya en "Las Mil y Una Noches" se encuentra una escena que refleja el tratamiento reclusivo que durante siglos ha venido sufriendo el paciente mental: "... mandó el rey Gayur que le pusiesen a su hija Budur sendos grilletes en los pies, esposas en las manos y al cuello una pesada cadena de hierro y de esa forma la atasen a la reja de su aposento. "También diferentes expresiones relacionadas con el tema han usado como vehículo la literatura: en la Edad Media, Jorge Manrique habla del amor como "un modo de locural con las mudanzas que hace:/ una vez pone tristura,/ otra vez causa holgura", reforzando así este modo de locura como un rasgo del amor cortés, que fuerza a la razón, y poniendo un referente de la conocida expresión utilizada hasta nuestros días.

Más conocida es la obra de Cervantes "El Quijote" en la que se nos muestra cómo es su protagonista, el loco más ilustre quizás de la historia de la literatura, objeto de burla e incluso de bromas pesadas, al igual que "El licenciado Vidriera", del mismo autor, a quien "los muchachos (...) le comenzaron a tirar trapos y aun piedras, por ver si era de vidrio, como él decía ". De esta misma época, el s. XVII, es el anónimo "Baile de los Locos de Toledo", en el que para disfrute del público los locos "cantan y bailan lo siguiente, $y$ en acabando sale el loquero con un azote."

La enfermedad mental se ha utilizado también en la literatura como un reflejo de las inquietudes del escritor sobre este tema. Dostoievski, enfermo de epilepsia, usa esta enfermedad como rasgo de algunos de sus personajes. En boca de uno de ellos dice que "... cualquier persona que contemple un ataque de epilepsia pensaría que era otro hombre dentro del que se veía, el que gritaba ". De este mismo autor, "El Jugador" refleja sus experiencias de ludópata. Luigi Pirandello, quien en su obra refleja la angustia ante el trastorno mental de su mujer, en "Cada cual c su juego" hace referencia a la doble personalidad.

Se puede observar, por tanto, como la narrative refleja de alguna manera la imagen de la enfermedac mental en sus contenidos y los mensajes que emite. con influencia en las creencias de salud de la población. Aun en obras separadas por un largo período de tiempo, se puede observar como el desconocimiento. las ideas erróneas y la utilización del "loco" comc refuerzo a la carga emocional de la historia puede ser una constante.

\section{MATERIAL Y MÉTODOS}

Las obras seleccionadas para el análisis de sus contenidos debían reunir los siguientes requisitos:

- desarrollar su acción en un centro de salud mental;

- tener en la lista de personajes a más de un paciente psiquiátrico;

- situadas en un contexto histrico que podía abarcar desde los años 50 hasta nuestros días.

La elección de estas características no es arbitraria, sino que se debe a una serie de criterios de interés ya que se verán reflejados en los resultados finales. Si el texto posee la primera característica se obtendrá información acerca de la vida de los pacientes en una institución psiquiátrica, la imagen de los tratamientos y cuidados de los que son objeto los pacientes, el concepto del hospital psiquiátrico que posee el autor y transmite a la sociedad... La segunda característica, además de aportar datos sobre la forma de relacionarse los pacientes y aumentar la lista de mensajes e ideas sobre el enfermo mental, evita que se recojan datos acerca de un único estereotipo de paciente: por ejemplo, son numerosas las obras que utilizan la figura de un psicótico como personaje central de una obra de terror o suspense ("El Resplandor", "El Alienista"...) pudiendo condicionar en gran medida los resultados obtenidos. Por último, la elección de un marco temporal se debe a que será más probable que la población relacione los mensajes emitidos por obras contemporáneas con la situación actual de la enfermedad mental (tratamientos, cuidados, pacientes...) que tome como todavía presentes situaciones descritas en un contexto mucho anterior en el tiempo. 
De la búsqueda bibliográfica llevada a cabo en varias bibliotecas municipales de Alicante se encontraron cuatro obras que reunían las características anteriormente citadas: "Los renglones torcidos de Dios", de Torcuato Luca de Tena (1979)'; "Alguien voló sobre el nido del cuco", de Ken Kesey (1962); "Historia clínica", de Vicente Ortuño (1991) y "Los alcohólicos", de Jim Thompson (1953). Para abreviar, se hará referencia a los textos con las siguientes iniciales: RTD, AVNC, HC y ALC, respectivamente para cada una de los textos anteriormente citados. De cada uno de ellos se procedió al análisis de aquellos contenidos que hicieran referencia al enfermo mental, tanto en la forma de referirse a éste en general como en las descripciones individuales de cada uno de los personajes; los tratamientos; los cuidados impartidos a los pacientes, con especial interés en los conceptos que se transmiten sobre la figura de la enfermera/ o psiquiátrica/ o; y otros aspectos no previstos en un primer momento pero que por su mensaje pudieran ser relevantes.

\section{RESULTADOS}

Todos los textos que componen el material utilizan como contexto principal para el desenlace de su argumento un hospital de salud mental; tres de ellos un centro para enfermos mentales de todo tipo y uno de ellos (ALC), un hospital para el tratamiento de deshabituación alcohólica. El hecho del multiculturalismo en los textos, reflejado tanto en la diferencia en las fechas que fueron escritos, en la nacionalidad de su autor como en sus conocimientos acerca de salud mental no ha constituido un factor esencial que afecte notablemente en el grado de coincidencia o acuerdo entre los resultados principales de cada una de ellas. Sobre los conocimientos sobre la materia anteriormente mencionados, es importante decir que el autor de RTD pasó un tiempo ingresado voluntariamente en un manicomio para documentarse acerca de la vida y características de los pacientes, además de haber leído algunos textos de Psiquiatría con el mismo fin; el autor de AVNC no cita explícitamente el haberse documentado, pero en su dedicatoria hace referencia a un amigo que "le enseñó la cueva del dragón", en clara metáfora hacia el hospital psiquiá- trico; sobre HC basta con decir que su autor es psicólogo, hecho que se hará notar en el texto por hechos como el correcto uso de vocabulario específico de su profesión como en la ausencia de frases despectivas para hacer alusión a los pacientes.

Los resultados que a continuación se mencionan expresan los puntos en los que coinciden los títulos del material en el global de los mensajes emitidos en sus textos. De esta manera, al dejar de lado observaciones muy particulares que puedan aparecer en uno sólo de los títulos se evitará la generalización de fenómenos con escaso o nulo reflejo en el resto de las obras analizadas. En el Cuadro 1 puede observarse un resumen de los principales resultados. Para evitar errores de interpretación se incluyen algunas citas textuales extraídas de los libros analizados, para evitar así problemas de subjetividad o interpretativos al exponer los resultados.

\section{Acerca de la "locura"}

Una de las características más común que aparece en los cuatro libros es el uso de frases y expresiones despectivas para aludir al enfermo o a su enfermedad. Estos enunciados pueden ir desde el más puro insulto a la más común de las expresiones o chascarrillo. Las frases más crueles se hayan en RTD, en el que constituyen una constante a lo largo de la narración: "...tristes deshechos de la humanidad " (Luca de Tena, 1991:276), "... los locos son una terrible equivocación de la naturaleza; son las faltas de ortografía de Dios... pequeña colección de monstruos " (Luca de Tena, 1991: 45). Frases similares se pueden encontrar en AVNC: los pacientes crónicos los describe como "... los deshechos del Establecimiento" (Kesey, 1976: 23) o generaliza al decir "... los cacharros rotos de la humanidad" (kesey, 1976: 261). No tan frecuentes como en los anteriores son las frases de este tipo que podemos encontrar en ALC, aunque sí con la misma carga emocional: "... despojo manchado de vómito y con mirada enloquecida" (Thompson, 1987: 156), "... los alcohólicos son menos exquisitos aún que los mendigos" (Thompson, 1987: 74). En HC el tratamiento que hace su autor

1.- Las fechas hacen referencia al año en que fueron escritos los libros, no a las ediciones analizadas: para ello ver el apartado dedicado a la bibliografía. 


\section{8 - Cultura de los Cuidados}

para reférirse al paciente está alejado de cualquier signo de desprecio: no pasa de expresiones comunes tales como " pirado " (Ortuño, 1994: 27), " lunático " (Ortuño, 1994: 49) o " pandilla de orates " (Ortuño, 1994: 54), en ocasiones más bien relacionadas con el tono de comedia de la historia. Estas expresiones más "amables" también aparecen en el resto de obras (lunático, orate, loco...).

Otro rasgo significativo son las "clasificaciones" de enfermedades mentales que aparecen en los textos. En los RTD cita una " jerarquía de la malignidad " (Luca de Tena, 1991: 65), dividiéndola en " ... ruina de la inteligencia" y "... ruina de la voluntad", además de otra clasificación en la que incluye características y tópicos sobre cada una de las categorías diagnósticas enumeradas:" Maníacos... eufóricos; Deprimidos... pavorosa tristeza; Esquizofrénicos... absurdidad; Dementes... idiotas; Profundos o demenciados... menos inteligencia que los gatos; furiosos, gritan" RTD (Luca de Tena, 1991: 69). Kesey, en AVNC, divide a los pacientes en agudos y crónicos, y a éstos últimos como " Ambulantes... que pueden andar sólos si se les alimenta; Rodantes... silla de ruedas; y Vegetales" (Kesey, 1976: 23). En ALC no hay una categorización explícita de la enfermedad mental, en parte porque todos los pacientes son alcohólicos, pero utiliza algunos diagnósticos para describir la personalidad de algunos pacientes: así, un lobotomizado es "...un imbécil completo... un idiota irremediable " (Thompson, 1987: 18) y en otra paciente aplica dos diagnósticos muy diferentes, pudiendo incluso contradecirse : "... depresiva $y$ alcohólica" (Thompson, 1987: 43), diagnóstico que se aproxima bastante a las características que describe y "... psicópata además de alcohólica" (Thompson, 1987: 128), diagnóstico bastante arbitrario. Con menos trascendencia y mucho más correcta es la clasificación descrita en HC: neuróticos, psicóticos y varios.

A las frases y giros para referirse a la enfermedad y a las peculiares clasificaciones se unen unas no menos curiosas definiciones de esta. Así, en RTD se presenta la enfermedad mental como ")... la locura consistía en la sublimación patológica o en la descoyuntación exagerada y morbosa de unas tendencias previas que ya estaban latentes en el individuo cuando era sano?" (Luca de Tena, 1991: 125); en AVNC se le dice a los pacientes que "... ustedes están aquí porque son incapaces de adaptarse a las normas sociales del Mundo Exterior,... porque han intentado esquivarlas y escapar a ellas... es posible que esa imprudente tolerancia de sus padres sea el germen que provocó su presente enfermedad" (Kesey, 1976: 221); en HC "... procesos mentales que, por vías todavía no suficientemente explicadas, afectaban las funciones cerebrales" (Ortuño, 1994: 127), y sobre el alcoholismo, en ALC se dice que "Todos saben que el alcohol sube por la espina dorsal hasta el cerebro, donde daña cada vez con mayor fuerza las frágiles células hasta que por fin las hace estallar y el interfecto se convierte en un auténtico imbécil." (Thompson, 1987: 25), por citar algunos ejemplos.

\section{El paciente: agresividad y características físicas}

Un factor común en los cuatro libros es el tópico estereotipo del paciente mental como individuo agresivo. Cuantitativamente los resultados obtenidos son los siguientes: en RTD, una proporción de 0'38 pacientes presentan actitudes agresivas; en AVNC, un 0'42; en HC, 0'41 y en ALC 0'14. Esta agresividad abarca desde la violencia física a la sexual.

También son abundantes las taras físicas en los pacientes cuando se aborda la descripción de los personajes. Igualmente expresado en proporciones, se encuentra en RTD un 0'34, en AVNC un 0'42, para ALC un 0'28 y 0 en HC. Estos datos aluden a los defectos puramente físicos, ya que el uso de ropas extravagantes o inadecuadas apenas tiene relevancia en parte debido al uso de ropa del hospital. Algunas de las taras resultan inverosímiles, como en el caso de RTD cuando describe a "la Mujer Percha": "Carece de espina dorsal. si la dejáramos caer se encogería como un acordeón y su cabeza se uniría con sus caderas" (Luca de Tena, 1991: 278). Estas descripciones no sólo se limitan a la descripción de los personajes sino que en ocasiones generaliza la anormalidad como rasgo inherente a la "locura": "...los morros abultados, las frentes minúsculas, la bocas abiertas y carnosas, las orejas voladoras, los pómulos mongólicos -cuando no los cuerpos deformes- abundaban en la comunidad" (Luca de Tena, 1991: 96); "... débiles, raquíticas, amedrentadas criaturas de una raza canija" (Kesey, 1976: 80); "Doc los miró uno a uno, ponderándolos men- 
talmente y encontrándolos horribles y llenos de defectos" (Thompson, 1987: 131).

\section{Tratamientos, cuidados y cuidadores}

Los textos analizados mencionan en ocasiones los tratamientos y actos médicos empleados como acciones con cierto grado de crueldad al abordar la descripción de estos: choques insulínicos, electroshocks, lobotomías y medicación "agresiva" son los tratamientos que aparecen (ver cuadro 1). De todos estos se destacan sin excepción aspectos negativos y equivocados. Por citar ejemplos de cada uno de ellos, el electroshock, elemento más explotado en AVNC es "Un artilugio que es como un compendio de la pastilla para dormir, la silla eléctrica y el potro de torturas, todo en uno. Es un buen truco, simple y apenas doloroso... Atan al paciente a una mesa con los brazos en cruz y con una corona de electrodos en vez de espinas " (Kesey, 1976: 83) " de sus palmas se desprendía una voluta de humo, producto de la corriente" (Kesey, 1976: 199) "... una víctima está sufriendo su tratamiento tras la puesta; puedo oír sus gritos" (Kesey, 1976: 208); sobre la medicación, en ALC un paciente relata cómo "... el corazón se me paraba... me encontraba tan incómodo e intranquilo que todo lo que deseaba era morirme" (Thompson, 1987: 50). Similares comentarios aparecen en la descripción del choque insulínico "... llevarla al borde mismo de la muerte provocándole una hipoglucemia progresiva hasta que entre en coma" (Luca de Tena, 1991: 184) y la lobotomización, terapias ya en desuso. Por último, RTD, AVNC y ALC hacen referencia a las celdas o habitaciones de aislamiento como medida para castigar comportamientos censurables en los pacientes. En HC no hace alusión a este respecto, pero plantea como medida para un paciente " la reclusión permanente en un alejado cenobio" (Ortuño, 1994: 19).

En contraposición con la crueldad descrita en estas terapias aparecen otras alternativas no tan "peligrosas" (ver Cuadro 1).

Otra constante en los cuatro textos, sin excepción, son las situaciones violentas provocadas por las enfermeras y el personal auxiliar. La proporción de cuidadores con actitudes violentas para cada uno de los textos es la siguiente: RTD, 0'60; AVNC, 0'50; ALC, 0'33; HC, 1. Datos tan significativos merecen un comentario. En HC sólo aparece una enfermera (llamada Killer), cuya agresividad se limita a "capones" y gritos, aunque describe sus "preferencias terapéuticas" de esta manera: "sacarles de la cabeza esas tonterías a base de métodos tradicionales y contrastados por la experiencia como la ducha fría, la camisa de fuerza y el ayuno forzoso, entre otros" (Ortuño, 1994: 42). En RTD las agresiones se manifiestan con amenazas verbales. En AVNC, la violencia ejercida por los cuidadores aparece en todos sus grados: mientras los celadores agreden física y verbalmente a los internos, la Gran Enfermera prescribe castigos tales como sesiones de electroshock o lobotomización a aquellos pacientes que no se adhieran a las normas fijadas por ella. En ALC, para la enfermera del hospital "No transcurre un día sin que ella tuviera su interludio deleitante en un momento $u$ otro. A veces no había sido nada más que un párpado retorcido bajo su pulgar profesional e inquisitivo. $O$ un caldo hirviendo embutido a la fuerza por entre unos labios demasiado débiles para protestar" (Thompson, 1987: 29): pero a pesar de todo "... era mejor que cualquier otra enfermera de los establecimientos que habian frecuentado anteriormente" (Thompson, 1987: 74).

A esto último se le unen comentarios que destacan la naturaleza "caritativa" o "vocacional" de los cuidados. A este respecto RTD se refiere a las cuidadoras/ es en estos términos: "No necesitó hacer estas visitas para confirmar las extraordinarias cualidades humanas de aquellas gentes" (Luca de Tena, 1991: 318), tal vez en alusión. a las "...mil faenas que eran más terribles de ver que fáciles de explicar" (Luca de Tena, 1991: 276). A pesar del carácter autoritario de su enfermera, HC aclara que "...sentía por los pacientes un cariño que no le impedía tratarlos como se merecían" (Ortuño, 1994: 41). La crueldad manifestada por la enfermera de AVNC contrasta con "las otras obras de caridad que realiza en la ciudad" (Kesey, 1976: 74). La actitud sádica de la enfermera de ALC es justificada por un paciente en estos términos: "... debe ser un suplicio tratar con borrachos un día tras otro. En realidad no se puede culpar a una persona porque se ponga dura y tosca" (Thompson, 1987: 74). 


\section{DISCUSIÓN}

Tras el análisis de los datos obtenidos tras la lectura de cada uno de los textos que componen el material, destaca el grado de coincidencia entre los resultados de cada uno de ellos. Esto es más notable si hacemos referencia al interculturalismo ya descrito que contextualiza y condiciona el contenido de las obras. En este sentido, es importante señalar que en $\mathrm{HC}$ la formación y conocimientos del autor sobre el tema tratado evita una visión tan "catastrofista" de la enfermedad mental: cierto es que los estereotipos y tópicos empleados en los otros libros aparecen también aquí, pero no causan una impresión tan violenta como en los otros, ya que en muchas ocasiones constituyen un recurso para dotar a la historia de su pretendido tono de comedia.

La imagen del enfermo mental como persona violenta e imprevisible en su comportamiento que se ofrece puede condicionar el carácter violento de los cuidadores, como una manera de imponer "la ley del más fuerte". La conducta destructiva dirigida hacia el exterior es relativamente rara en las enfermedades mentales. El miedo con el que algunas personas miran a los pacientes psiquiátricos es completamente desproporcionado con el grupo más bien pequeño que representa un peligro para los demás (Kaplan y Sadock,1991: 599). A pesar de ello la imagen ofrecida por distintos medios sobre el paciente psiquiátrico a este respecto es precisamente la que yace en la conciencia de la población general: las noticias acerca de delitos cometidos por personas enfermas permanecen varios días en titulares, en contraposición a las que aluden a éxitos terapéuticos en el terreno de la reinserción, que son prácticamente nula. No es de extrañar, por tanto, que dicho estereotipo constituya un auténtico filón, cada vez más explotado, para guiones cinematográficos u otros textos, con lo cual se continúa reforzando todavía más este tipo de aspectos negativos.

Las referencias a la crueldad de los procedimientos terapéuticos empleados en Psiquiatría puede tener dos explicaciones: la falta de conocimientos o información errónea, o que estas constituyen un recurso para aumentar la carga emocional del relato. Esto salta a la vista, por ejemplo, en la manera en la que se trata al "electroshock": en primer lugar decir que esta denominación por sí misma ya tiene unas connotaciones negativas es más común referirla como Terapia Electro- Convulsiva (T.E.C.) El riesgo de muerte es menor por TEC que por antidepresivos y su efectividad está fuera de toda duda, teniendo hoy día indicaciones terapéuticas claras (Santiuste et al., 1993: 44). Y, desde luego, no se emplea como castigo ni los pacientes "echan humo" tras su aplicación. También se habla de unidades de aislamiento que en varias ocasiones también son empleadas como castigo o represalia hacia los pacientes. Esta creencia, también errónea, está bastante extendida, tal como se refleja en los resultados. Hay que decir que el aislamiento o sujeción del paciente en otra medida terapéutica más que tiene sus indicaciones: para evitar autolesiones en pacientes agitados o para la restricción de estímulos externos en estados hipermaníacos, por ejemplo. El uso apelativos tan comunes en la población para referirse al enfermo mental que se emplean en los diversos textos, así como otro tipo de frases hechas ("... se cuenta que los niños y los locos dicen siempre la verdad ") (Luca de Tena, 1991: 288) demuestra que el tipo de material utilizado constituye un medio de difusión para este tipo de expresiones. Pero lo todavía más significativo es que haga uso de frases insultantes o degradantes, generalizándolas en muchas ocasiones no a personajes concretos sino a todo el colectivo de pacientes. Frases de este último tipo hacen dan una imagen de personas inútiles que incluso constituyen una carga para la sociedad. No es de extrañar que sobre este precepto se justifique la idea de unos cuidados de tipo caritativo, de medidas represivas contra los pacientes, del trato autoritario (que no se daría a pacientes físicos)... que tanto aparece en los textos. Aunque también aparecen aspectos positivos, como en el caso de algunas acciones terapéuticas, estas quedan eclipsadas por la totalidad de mensajes negativos emitidos, tanto por su cantidad como por el impacto emocional que supone para el lector.

\section{CONCLUSIONES}

- El grado de coincidencia entre los resultados obtenidos en cada uno de los libros analizados muestra una visión negativa del enfermo mental y de su entorno bastante extendida en la sociedad. Este hecho se debe no sólo a las creencias del propio autor, sino también a que éste refleja en la narración el miedo y el desconocimiento que tiene la sociedad sobre este tema.

- El hecho de que esta coincidencia sea a nivel de mensajes negativos y peyorativos confirma la hipóte- 
sis planteada inicialmente. Para esto hay que tener en zuenta la influencia que tiene sobre los conocimien:os y creencias sobre la salud los mensajes que a este respecto recibe la sociedad, hecho también planteado al principio.

- Si es evidente la existencia de una imagen negativa hacia el enfermo mental por parte de la sociedad, alimentada por creencias erróneas, será función del profesional de la salud la identificación de ideas equivocadas que constituyan un elemento ansiógeno tanto en el paciente como en su familia.

- Coincidiendo con Siles et al. (1993), que emplearon una metodología similar, esta propuesta de utilización de textos como modelos alternativos en la investigación parece de utilidad una vez vistos los resultados obtenidos, además de las ventajas que ofrece: es un medio económico en comparación con otros; se obtienen resultados a nivel de contenidos, lo que permite averiguar el grado de importancia del problema a través de los mensajes aparecidos en diálogos y descripciones; es un material fácilmente accesible a otros profesionales que estén interesados en rebatir o profundizar en los resultados; y puede ser utilizado para la realización de prácticas simuladas. Coincidiendo también con Hereile et al. (1992), quienes centraron su estudio en el análisis de textos periodísticos, la técnica de análisis de contenido puede ser una herramienta buena para analizar y planificar estrategias de información a la prensa.

- Este trabajo sólo muestra una parte de la realidad que forma el campo de elementos relacionados sobre la imagen del enfermo y la enfermedad mental. Dada la importancia que tiene tanto para la imagen como para la integración del paciente, se estima conveniente continuar el estudio de la naturaleza y calidad de estos mensajes en otros medios de comunicación (prensa, televisión...), así como una aproximación más directa a las creencias de la población en concreto, todo ello para delimitar en sentido más amplio este problema y plantear a otros niveles estrategias de acción (información).

\section{BIBLIOGRAFÍA}

Cabrera Forneiro, J. (1995) Esquizofrenia y su independencia familiar. Rev Minusval. Ministerio de Asuntos Sociales (INSERSO), 95: 43 .
Hereile Valero, C; Quero Rufián, A.; Calzada Gómez, M.L. (1992) La salud vista por la prensa. Rev Rol, 170: 79- 82.

Isla Pera, P; López Matheu, C; Pedreny, R; Guirbau, M.R.; Domenech, E. (1995) Publicidad, salud y educación sanitaria. Rev Rol, 201: 59-63.

Kaplan, H.I.; Sadock, B.J (1991) Urgencias psiquiátricas. En Compendio de Psiquiatría. Ed. Masson- Salvat Medicina. $2^{\mathrm{a}}$ ed. Barcelona: 592- 612 .

Kesey, K. (1976) Alguien voló sobre el nido del cuco. Argos. $1^{a}$ ed. Barcelona.

Luca de Tena, T. (1991) Los renglones torcidos de Dios. Planeta. $33^{\mathrm{a}}$ ed. Barcelona.

Mauleón García, M.A. (1993) Enfermería ante el paciente psiquiátrico. Rev Rol, 177: 34-38.

Martí Tusquets, J.L.; Murcia Grau, M. J. (1988) Planificación de la asistencia psiquiátrica. En Enfermedad Mental y Entorno Urbano. Anthropos. Barcelona.

Molina, F.; De La Hoz Garcimartín, M.A.; De La Cruz López, I. (1994) Esquizofrenia. )Qué saben los familiares?. Rev Rol, 189: 25-29.

Ortiz Viudes, I; Pascual Martínez, R; Sánchez Parra, J. (1995) Esquizofrenia simple. Plan de cuidados de Enfermería. Enfermería Científica, 162163: 72-76.

Ortuño, V. (1991) Historia clínica. Aguaclara. 1ª ed. Alicante.

Rubio, V.J. (1994) Aspectos psicológicos y sociales de las plurideficiencias. Rev Minusval. Ministerio de Asuntos Sociales (INSERSO), 92: 24-26.

Santiuste de Pablos, M.; Salorio del Moral, P.; Flores Biernet, M.D.; Ortiz, A. (1993) Terapia electroconvulsiva. Actuación del personal de Enfermería. Enfermería Científica, 131: 44- 48.

Siles, J.; Fernández, P.; Pérez, R.M.; García, E. (1993) Las alteraciones en la vida cotidiana de enfermos terminales a través del análisis de textos. Un modelo simulado para las prácticas con el proceso de Enfermería. Enf Científica, 131: 4- 9.

Thompson, J. (1987) Los alcohólicos. Júcar. $1^{a}$ ed. Barcelona. 


\section{CUADRO 1}

RESULTADOS PRINCIPALES

\begin{tabular}{|c|c|c|c|c|}
\hline & $\begin{array}{l}\text { Los renglones } \\
\text { torcidos de Dios } \\
\text { (RTD) }\end{array}$ & $\begin{array}{l}\text { Alguien voló } \\
\text { sobre el nido del } \\
\text { cuco (AVNC) }\end{array}$ & $\begin{array}{l}\text { Historia clínica } \\
\text { (HC) }\end{array}$ & $\begin{array}{l}\text { Los alcohólicos } \\
\text { (ALC) }\end{array}$ \\
\hline $\begin{array}{l}\text { Uso de frases } \\
\text { despectivas }\end{array}$ & +++ & +++ & + & ++ \\
\hline $\begin{array}{l}\text { Pacientes } \\
\text { agresivos }\end{array}$ & 0,38 & 0,42 & 0,41 & 0,14 \\
\hline $\begin{array}{l}\text { Defectos } \\
\text { físicos en } \\
\text { pacientes }\end{array}$ & 0,34 & 0,42 & 0 & 0,28 \\
\hline $\begin{array}{l}\text { Tratamientos } \\
\text { crueles }\end{array}$ & $\begin{array}{l}\text { Shock insulínico, } \\
\text { electroshock, } \\
\text { habitaciones de } \\
\text { castigo }\end{array}$ & $\begin{array}{l}\text { Electroshock, } \\
\text { lobotomía, } \\
\text { medicación, } \\
\text { habitaciones de } \\
\text { castigo, duchas a } \\
\text { presion }\end{array}$ & $\begin{array}{l}\text { Lobotomía, } \\
\text { electroshock, } \\
\text { "cócteles de } \\
\text { sedantes" }\end{array}$ & $\begin{array}{l}\text { Lobotomía, } \\
\text { medicación, } \\
\text { aislamiento }\end{array}$ \\
\hline $\begin{array}{l}\text { Cuidados y } \\
\text { terapias no } \\
\text { agresivas }\end{array}$ & $\begin{array}{l}\text { Tests, EEG, T.A., } \\
\text { ejercicio físico, } \\
\text { excursiones, tests, } \\
\text { tareas de } \\
\text { mantenimiento, } \\
\text { atención a } \\
\text { pacientes } \\
\text { dependientes }\end{array}$ & $\begin{array}{l}\text { Sesiones de grupo, } \\
\text { ejercicio físico, } \\
\text { juegos en grupo, } \\
\text { excursiones, tests, } \\
\text { tareas de } \\
\text { mantenimiento, } \\
\text { atención a } \\
\text { pacientes } \\
\text { dependientes }\end{array}$ & $\begin{array}{l}\text { Laborterapia, } \\
\text { tests, ejercicio } \\
\text { físico, } \\
\text { psicoterapia, } \\
\text { salidas al cine, } \\
\text { tareas de } \\
\text { mantenimiento }\end{array}$ & $\begin{array}{l}\text { Psicoterapia, } \\
\text { atención a } \\
\text { pacientes } \\
\text { dependientes }\end{array}$ \\
\hline $\begin{array}{l}\text { Actitudes } \\
\text { agresivas en } \\
\text { cuidadores }\end{array}$ & 0,60 & 0,50 & 1 & 0,33 \\
\hline
\end{tabular}

+++: muy significativo; ++: significativo; +: escasamente/nada significativo. Los datos numéricos se expresan como proporciones 